\title{
Slow Relaxation of Spin-Cast Poly(methyl methacrylate) Confined in Thin Films
}

Helen Richardson, Michele Sferrazza and Joseph L. Keddie

Department of Physics, University of Surrey, Guildford, Surrey GU2 7XH, UK

\begin{abstract}
Although the volume relaxation of bulk homopolymer glasses is thoroughly understood, many questions remain about the volume relaxation of polymers cast from solvent. Furthermore, in polymer thin films, the possible effects of confinement, surfaces and interfaces on relaxation are largely unexplored. Measurements of the film thickness of spin-cast poly(methyl methacrylate) thin films over extended periods of time, using spectroscopic ellipsometry, reveal that the characteristic time for relaxation increases with film thickness. This result is consistent with the idea of enhanced molecular mobility in thin films, and it might reflect the conformation and a reduced extent of entanglements of the polymers when spin-cast from dilute solution.
\end{abstract}

\section{INTRODUCTION}

Understanding structural relaxation in polymers is important for predicting long-term changes in their physical properties, such as bulk and shear moduli, density, refractive index and dielectric strength. Indeed, there exists a vast body of literature describing relaxation behavior in bulk systems [1-4], but to-date relatively few studies have been carried out on polymers confined in thin films [5]. Of these studies, most have concerned films cooled from the polymer melt and very few have concerned the relaxation of unannealed spin-cast polymer films, although it has been recognized that these are metastable forms of soft matter of scientific interest [6].

A question of fundamental importance is whether the dynamics of relaxation in confined polymers is the same as in bulk polymers [7]. The modes of relaxation in a polymer near an interface with a substrate or the atmosphere are likely to differ from those modes in the bulk [5]. Hence, the distribution of relaxation times is likewise expected to differ. Moreover, studying the relaxation behaviour in unannealed, spin-cast films is potentially very useful because it may help to explain a number of phenomena such as de-wetting [8], drying [9] and stress relaxation [10].

Structural relaxation in thin films is difficult to study experimentally. Volume changes during the relaxation of a polymer glass are typically of the order of 1 part in $10^{4}$ [3,4], and so a non-invasive experimental technique for studying thin films must be sensitive to dimensional changes of less than $1 \mathrm{~nm}$. The technique must be fast enough to detect thickness changes occurring over a few minutes, while it must also be stable enough to observe changes occurring over several days. Neutron [11] and X-ray [12] reflection have been shown to have sufficient resolution to probe small dimensional changes in thin films. They are thus appropriate techniques for this type of experiment. Spectroscopic ellipsometry, which likewise offers sub$\mathrm{nm}$ resolution of film thickness [13], can obtain measurements in less than a minute but can also monitor changes over extended periods. In our work, we therefore use ellipsometry as a noninvasive probe of dimensional change in thin films.

The analysis of unannealed, freshly-cast thin films adds another layer of complexity, because there is solvent loss during the relaxation [6,9]. A complementary technique is required to probe the mass change simultaneously, so that structural relaxation and solvent loss can be 
examined independently. In previous work [14], we have used a quartz crystal microbalance to detect mass changes of the order of nanograms in spin-cast polymer thin films.

Poly(methyl methacrylate) (PMMA) is one of several homopolymers for which a body of bulk relaxation data exists [3]. In a typical experiment, the time-dependent volume $V_{t}$ of a glass after a quench from the melt is measured. An equilibrium volume, $V_{\infty}$, is also found from the value obtained when the glass has fully relaxed; it represents the volume that would be obtained in a liquid cooled infinitely slowly without passing through the glass transition. A function to describe the departure from equilibrium $\delta$ is defined as $\delta=\left(V_{t}-V_{\infty}\right) / V_{\infty}[1,4]$. A volume relaxation rate $\beta$ is then obtained from the gradient of a plot of $\delta$ as a function of the logarithm of time, $t$, given as $\mathrm{d} \delta / \mathrm{d}(\log t)$. For bulk PMMA that has been quenched from the melt at $120{ }^{\circ} \mathrm{C}$ to a glass at $60{ }^{\circ} \mathrm{C}, \beta$ has been found to be $c a .4 \times 10^{-4}$ [3]. Values of $\beta$ can be used to compare two polymers or to examine the effect of temperature and thermal history. A limitation of this type of analysis, however, is that it cannot distinguish a distribution of relaxation times from a timedependent $\delta$ [4].

A glass's relaxation function $\phi$ cannot usually be described by a single exponential function. In an important development in the field, Kohlraush, Williams and Watts (KWW) [15] proposed the use of a stretched-exponential function to consider a distribution of relaxation times, where $\phi$ is given as $\exp \left[-(t / \tau)^{v}\right]$. Here, the relaxation is characterised by a relaxation time $\tau$ and by an exponent $v$, which is inversely proportional to the width of the distribution of relaxation times. A value of 1 represents a single distribution time, whereas lower values represent wider distributions of times. For volume relaxation, $\phi$ is defined as $\left(V_{t}-V_{\infty}\right) /\left(V_{0}-V_{\infty}\right)$, where $V_{0}$ is the initial volume after a thermal quench [4]. Thus, $\phi$ varies from 1 to 0.

During the spin-casting process, vitrification occurs when enough solvent has evaporated to raise the glass transition temperature $\left(T_{g}\right)$ of the solution to the temperature of the experiment $[6,16]$. The glass transition occurs as a result of a concentration increase rather than a temperature decrease. A certain concentration of solvent is present in the glass as it passes through the transition. Formation of micro- air voids in the glass with further solvent loss is thermodynamically unfavourable [6], and so solvent loss is accompanied by a decrease in volume of the glassy matrix, which is achieved by volume relaxation. In this way, the rate of solvent leaving a glass is linked to its initial relaxation dynamics. In polymers with a high $T_{g}$, a large fraction of solvent will be initially confined in the glass [14,16], and hence the volume change during relaxation will be significant.

\section{EXPERIMENTAL DETAILS}

PMMA with a polydispersity $M_{w} / M_{n}=1.01$ and an average $M_{w}=1.42 \times 10^{5} \mathrm{~g} \mathrm{~mol}^{-1}$ was obtained from Polymer Laboratories and used as received. Its $T_{g}$ was determined by differential scanning calorimetry to be $109{ }^{\circ} \mathrm{C}$. Polymer films were spin-cast from dilute solutions in toluene onto (100) silicon substrates using a spin time of 3 sec. and a spin speed of $2000 \mathrm{rpm}$. Film thickness as a function of time was determined using a rotating-analyzer spectroscopic ellipsometer (J.A. Woollam. Inc., USA). To control the temperature, samples were mounted on a heating stage (Linkam, Surrey) placed in the ellipsometer beam. At the start of each experiment, ellipsometry parameters ( $\psi$ and $\Delta$ ) were acquired at four different wavelengths as a function of time at intervals of about 20 sec., as described elsewhere [14]. When the rate of thickness change became slow, this type of dynamic scan was not necessary, and instead spectroscopic 
scans over the wavelength range 400 to $800 \mathrm{~nm}$ at intervals of $5 \mathrm{~nm}$ were carried out every 30 $\min$.

\section{RESULTS AND DISCUSSION}

A significant decrease in film thickness was observed at room temperature (RT) in spincast films, and a further decrease occurred after annealing above the $T_{g}$ and cooling to room temperature. As an example, Figure 1 shows ellipsometry spectra ( $\psi$ and $\Delta$ ) for a PMMA film at RT taken $1 \mathrm{hr}$. and 4 days after spin-casting. Spectra from the same film after annealing at 150 ${ }^{\circ} \mathrm{C}$ are shown for comparison. The shifts in the spectra are much greater than the experimental uncertainty in the data points. The dispersion of the optical constants of the PMMA can be described by a Cauchy equation [17], and then the film thickness $h$ is obtained from the best-fit of the data to a model. Over the four days, $h$ decreased from $163.7 \mathrm{~nm}$ to $154.0 \mathrm{~nm}$, and after annealing, it decreased further to $149.3 \mathrm{~nm}$, corresponding to a total change of nearly $9 \%$.

Using dynamic ellipsometry scans, $h$ of this film was monitored over time, as presented in Figure 2. The data show a rapid thickness decrease over the first few hundred minutes, followed by a further decrease in thickness over 7 days or more after spin-casting. Our previous work using quartz crystal microgravimetry [8] has indicated that, the decrease in the thickness of PMMA films is accompanied by a simultaneous loss of solvent. However, since the rate of decrease is many orders of magnitude lower than that expected from the solvent diffusion coefficients in the literature [8], we conclude that the rate of decrease is governed by the relaxation of the glassy polymer-solvent matrix. Solvent does not leave the glass without simultaneous volume relaxation.

The inset figure plots the same data in the conventional way that volume relaxation data are often presented in the literature [1-4]. Also included are data for a freshly spin-cast $150 \mathrm{~nm}$ PMMA film being annealed at $55^{\circ} \mathrm{C}$. We define $\delta$ in terms of $h_{t}$ rather than $V_{t}$. The value for $h_{\infty}$ was the thickness after annealing for $10 \mathrm{~min}$. and cooling at $2{ }^{\circ} \mathrm{C} \mathrm{min} .^{-1}$ to the initial temperature of either $25^{\circ} \mathrm{C}$ or $55^{\circ} \mathrm{C}$. It is apparent that the time-dependence of the thickness decrease does not vary strongly with temperature. $\beta$, determined from the slope of each curve, was found to be $2.5 \times 10^{-2}$ and $2.7 \times 10^{-2}$ for the films at $25^{\circ} \mathrm{C}$ and $55^{\circ} \mathrm{C}$, respectively. These are about two orders of magnitude higher than the previously reported values of ca. $4.0 \times 10^{-4}$ for bulk PMMA

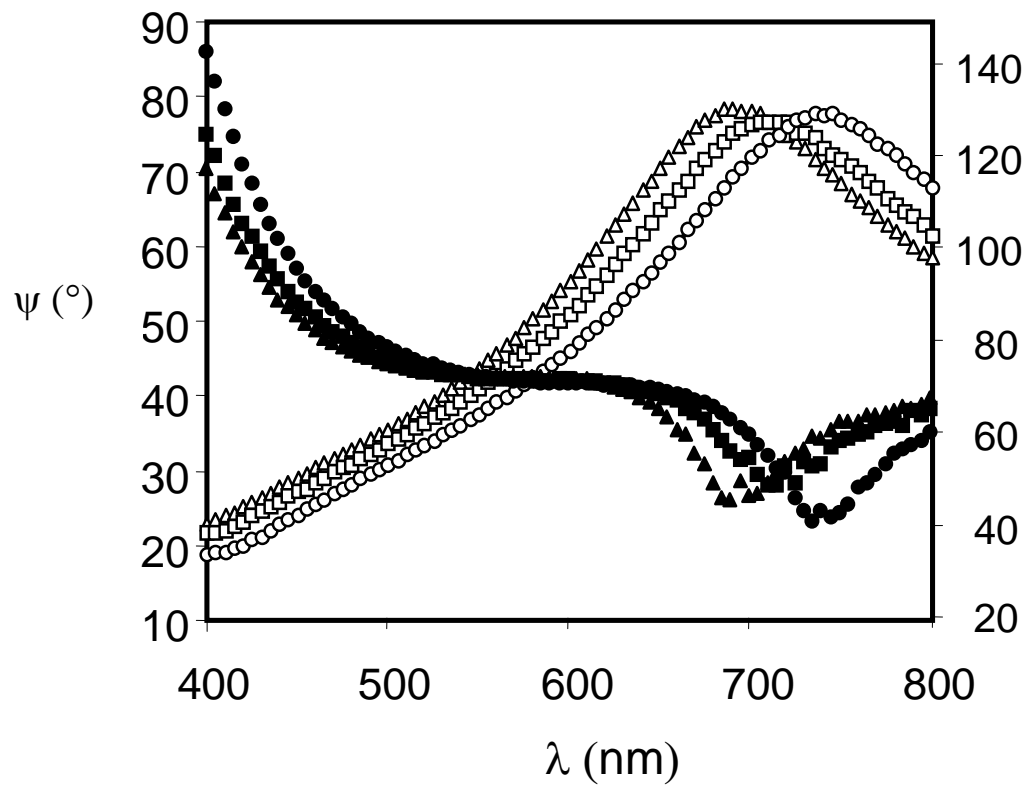

Figure 1. Ellipsometry spectra showing $\psi$ (unfilled symbols) and $\Delta$ (filled symbols) for a PMMA film on Si at RT: 1 hr. after spincasting (circles); 4 days after spin-casting (squares); and after annealing at $150{ }^{\circ} \mathrm{C}$ for $10 \mathrm{~min}$ and cooling at $2{ }^{\circ} \mathrm{C}$ $\mathrm{min}^{-1}$ to RT (triangles). The angle-of-incidence was $72^{\circ}$. 


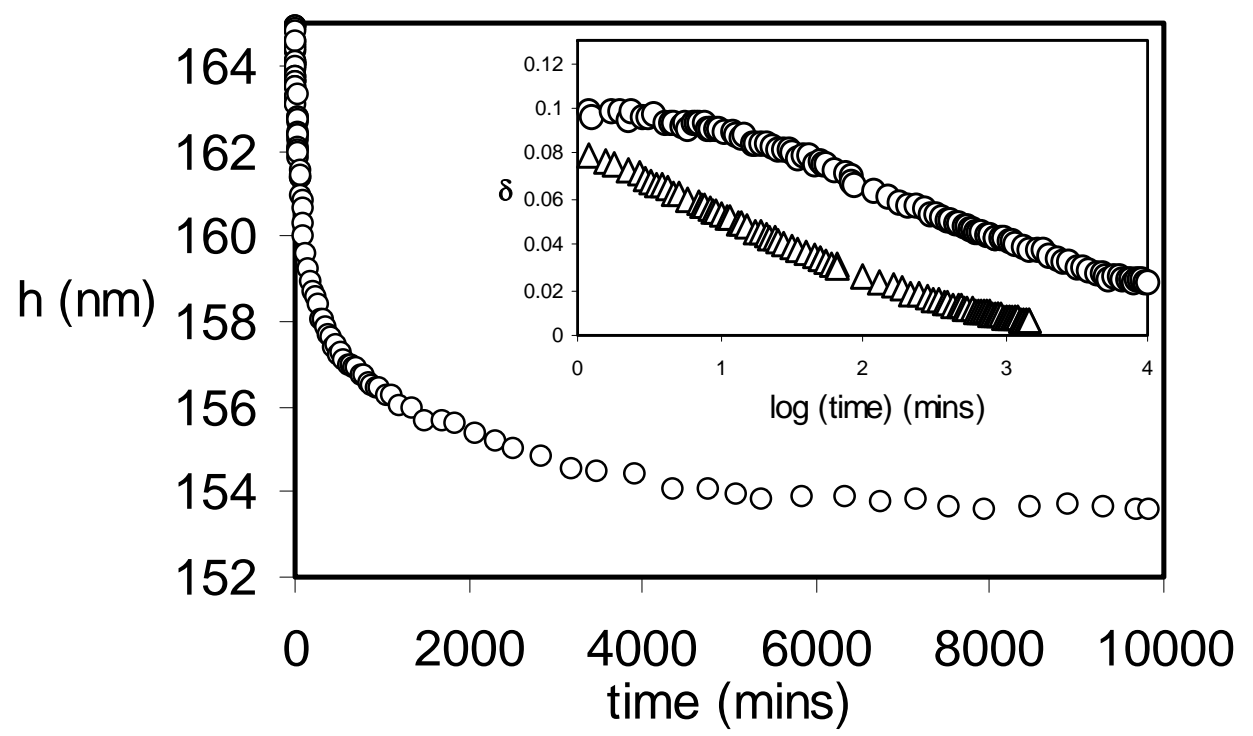

Figure 2. The continuous decrease in $h$ over time after spin-casting for the same PMMA film as shown in Figure 1. Inset: $\delta$ versus the logarithm of time for the same film (circles) compared to one of similar thickness at $55^{\circ} \mathrm{C}$ (triangles). The number of data points has been reduced for clarity.

quenched to $55^{\circ} \mathrm{C}$ from the melt [3]. Another major difference is that the initial value of $\delta$ for bulk PMMA is $\delta_{0}=0.016$, which is much smaller than the $\delta_{0} \approx 0.1$ observed in the spin-cast films. We are currently investigating the reasons for the large $\delta_{0}$ in the films as well as an observed dependence of $\delta_{0}$ on film thickness. It is relevant to note that Kovacs [1] has derived a relationship predicting that $\beta$ is proportional to $\delta$, which is consistent with the differences in $\beta$ found between bulk PMMA and spin-cast films.

Although the determination of $\beta$ is commonly reported in the relaxation literature [1-4], it has its limitations. As seen in Figure 2, the gradient of the data is changing over time, and so a single value of $\beta$ cannot be applied to the entire relaxation process. By comparison, the KWW analysis considers a distribution of relaxation times and is therefore better able to describe relaxation data. Additional experiments were performed at a temperature of $55^{\circ} \mathrm{C}$, because at lower temperatures the relaxations have exceedingly long time scales and cannot realistically be studied in experiments.

As an example of our KWW analysis, Figure 3 compares plots of $\phi$ for $31 \mathrm{~nm}$ and $256 \mathrm{~nm}$ PMMA films relaxing at $55{ }^{\circ} \mathrm{C}$ over a period of $1200 \mathrm{~min}$. (In this analysis, $h_{\infty}$ was obtained from the thickness value at $t=1200 \mathrm{~min}$.) The values of $v$, obtained from the best fit to the KWW equation for each data set, were found - within an experimental uncertainty of \pm 0.05 - to be the same for both films, i.e. between 0.4 and 0.5 . Incidentally, this value is similar to one obtained elsewhere via photon correlation spectroscopy for freely-standing films of PS [T]. $v$ is a measure of the distribution of relaxation times, and the invariance of $v$ with thickness suggests that, over the time scale studied, the distribution of molecular mobility is similar in both cases. 


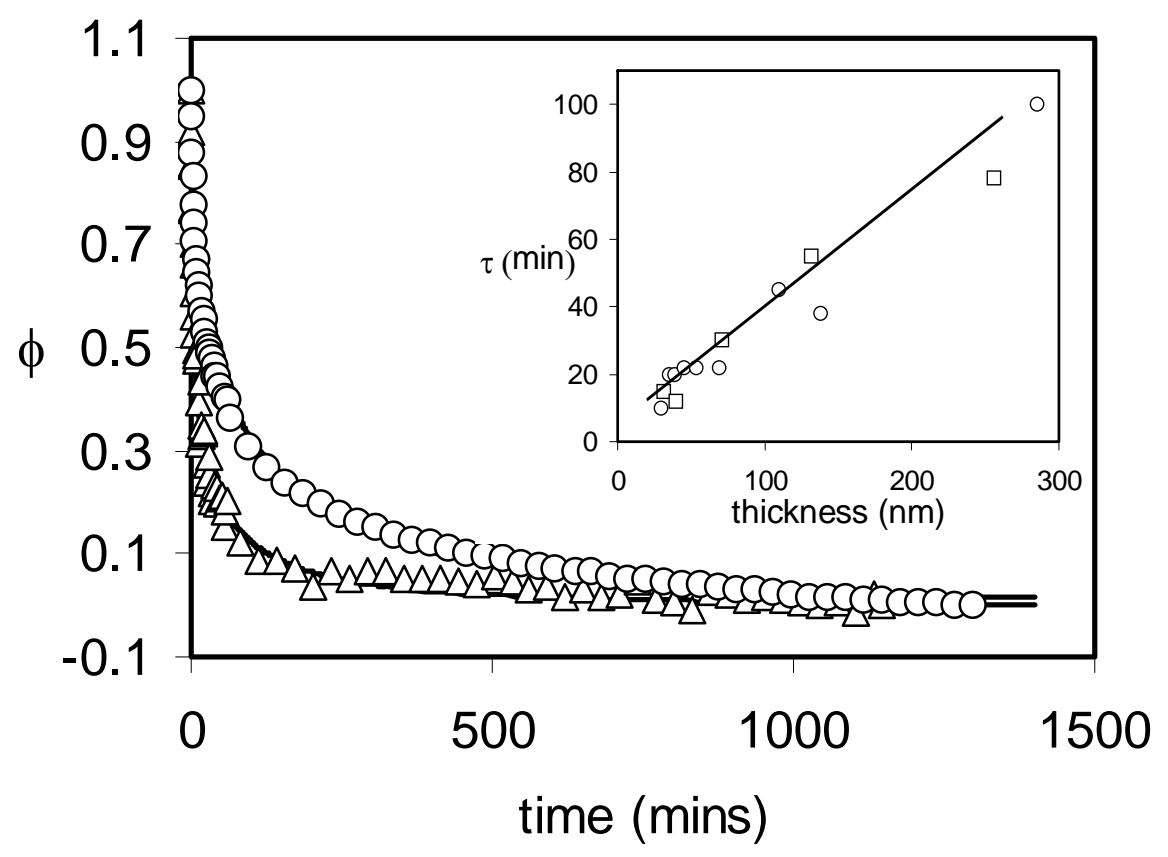

Figure 3. $\phi$ as a function of time for freshly-cast PMMA films of two different $h_{\infty}$ (obtained at $1200 \mathrm{~min}$ ): $31 \mathrm{~nm}$ (triangles) and 256nm (circles). Solid lines show the best fits to the KWW equation, using $v=0.4$ and $\tau=15 \mathrm{~min}$ (31 nm film); $v=0.5, \tau=78 \mathrm{~min}$ (256 nm film).

Inset: $\tau$ as a function of film thickness for PMMA films immediately after casting and heating to $55^{\circ} \mathrm{C}$ (squares), and conditioned for $1 \mathrm{hr}$ at $25^{\circ} \mathrm{C}$ before being heated to $55^{\circ} \mathrm{C}$ (circles).

The average characteristic relaxation time, $\tau$, on the other hand, was found to be higher for the thicker film: $78 \mathrm{~min}$. compared to $15 \mathrm{~min}$. A further illustration of the dependence of $\tau$ on thickness is given in the inset figure which shows $\tau$ for several films. In a film that is approximately $300 \mathrm{~nm}$ thick, $\tau$ approaches $100 \mathrm{~min}$. From data in the literature [3] for bulk PMMA at $60{ }^{\circ} \mathrm{C}$, we find $\tau=90 \mathrm{~min}$. and $v=0.55$, when $V_{\infty}$ is likewise determined from the volume at $1200 \mathrm{~min}$. Thus it appears that the relaxation of spin-cast thin films approaches the bulk behavior as thickness increases.

An obvious interpretation of this result is that molecules in thinner films have greater mobility. The phenomenon might not be the result of the effects of a free surface on molecular mobility per se, however, but it might instead be linked to the molecular conformation and the extent of entanglement in the spin-cast film. When a solvent is removed quickly from a polymer solution, it has been found elsewhere that the $T_{g}$ of the glass is reduced from the bulk value, and the magnitude of the reduction is inversely related to the original concentration [19]. One explanation for this result is that if the concentration of the solution is below the overlap concentration $\phi^{*}$ where the chains come into contact with each other, they will be less able to entangle when the solvent is removed quickly. Similarly, in the spin-casting process, most of the solvent evaporates very quickly, which might prevent chain entanglement in the glassy film. Our thicker films are cast from solutions with $\phi \approx 0.03$, which is in the overlap regime $\left(\phi>\phi^{*}\right)$. (For the molecular weight used, $\phi^{*}$ is estimated to be $2 \times 10^{-3}$ [20]). Thinner films, in contrast, are cast from more dilute solutions, in which the likelihood of entanglements might be reduced 
compared to what is found in solutions near $\phi^{*}$. If there is less entanglement in the thinner films, there would be fewer restrictions on molecular mobility and hence a faster relaxation. Recent work [21] has questioned whether the $T_{g}$ reduction in freeze-dried cyclic polymers is the result of a reduced entanglement density, however, and so further research on spin-cast films is required.

\section{CONCLUSIONS}

The volume relaxation in glassy polymer thin films prepared by "quenching” from solutions in solvent during the spin-casting process is a topic of fundamental importance that has been relatively neglected in the scientific literature. Significant film thickness changes taking place over days have been observed in spin-cast PMMA films using spectroscopic ellipsometry. This relaxation process can be well described using a KWW stretched-exponential function and fitting to a characteristic relaxation time, $\tau$. The value of $\tau$ is found to increase with the film thickness, and approaches a value that is consistent with the volume relaxation of the bulk homopolymer. We tentatively attribute this result to enhanced mobility in thinner films, because of a lower entanglement density resulting from spin-casting from more dilute solutions, below the overlap concentration.

\section{ACKNOWLEDGMENTS}

We are grateful to the UK's Engineering and Physical Sciences Research Council for funding.

\section{REFERENCES}

1. A.J. Kovacs, Fortschr. Hochpolym.-Forsch. 3, 394 (1964).

2. I. M. Hodge, J. Non-Cryst. Sol. 169, 211 (1994).

3. J.M. Hutchinson and C.B. Bucknall, Polym. Eng. Sci. 20, 173 (1980).

4. J.M. Hutchinson, Prog. Polym. Sci. 20, 703 (1995).

5. S. Kawana and R.A.L. Jones, Eur. Phys. J. E 10, 223 (2003).

6. G. Reiter and P.G. de Gennes, Eur. Phys. J. E 6, 25 (2001).

7. R.A.L. Jones, Curr. Opin. Coll. Interf. Sci. 4, 153 (1999).

8. H. Richardson, C. Carelli, J.L. Keddie and M. Sferrazza, Eur. Phys. J. E, to appear.

9. P.G. de Gennes, Eur. Phys. J. E 7, 31 (2002).

10. J.R. McLoughlin and A.V. Tobolsky, J. Polym. Sci. 6, 658 (1951).

11. T. Kanaya, T. Miyazaki, H. Watanabe et al., Polymer 44, 3769 (2003).

12. M. Mukherjee, M. Battacharya, M.K. Sanyal et al, Phys. Rev. E 66, 061801 (2002).

13. J.L. Keddie, Curr. Opin. Coll. Interf. Sci. 6, 102 (2001).

14. H. Richardson, M. Sferrazza and J.L. Keddie, Eur. Phys. J. E Direct, 12, s01, 012 (2003).

15. G. Williams and D.C. Watts, Trans. Faraday Soc. 66, 80 (1970).

16. G.B. McKenna, J. Phys. IV France 10, 53 (2000).

17. B. Parbhoo, S. Izrael, J.M. Salamanca, and J.L. Keddie, Surf. Interf. Anal. 29, 341 (2000).

18. J.A.Forrest, C.Svanberg, K.Revesz, M.Rodahl, L.M.Torell, and B.Kasemo, Phys. Rev. E 58, R1226 (1998).

19. D. Huang, Y. Yang, G. Zhuang, L. Binyao, Macromolecules 33, 461 (2000).

20. G. Strobl, The Physics of Polymers, $2^{\text {nd }}$ Ed., (Springer, Berlin, 1997) pp. 64-65.

21. S.L. Simon, P. Bernazzani, and G.B. McKenna, Polymer 44, 8025 (2003). 